\title{
La mezcla del marketing y la satisfacción del cliente en la Unidad Educativa Particular Redemptio, Jipijapa, Manabí, Ecuador.
}

\section{The marketing mix and customer satisfaction in the Unidad Educativa Particular Redemptio, Jipijapa, Manabí, Ecuador.}

La mezcla del marketing y la satisfacción del cliente

Jenny Santistevan Nunura ${ }^{(1)}$

Martha Escobar García (2)

(1) Universidad Técnica de Manabí, Ecuador. santistevanjenny@ hotmail.com

ORCID: https://orcid.org/0000-0002-9168-3411

(2) Universidad Técnica de Manabí. Ecuador.marthitae65@gmail.com

ORCID: https://orcid.org/0000-0002-1939-448X

Contacto: santistevanjenny@hotmail.com

\section{Resumen}

El objetivo de esta investigación fue medir el nivel de satisfacción del cliente respecto de la mezcla del marketing en la Unidad Educativa Particular "Redemptio" de la ciudad de Jipijapa de la Provincia de Manabí. La investigación es de tipo exploratoria descriptiva. El diseño fue no experimental y transeccional. La población fue de 408 representantes legales de la Unidad Educativa Particular Redemptio. La muestra de 199 padres de familia desde el Nivel Inicial hasta el Bachillerato General Unificado, se calculó con el 5\% de margen de error y el 95\% de nivel de confianza, en el sitio web de Asesoría Económica \& Marketing S. A. (2009). Se utilizó un cuestionario de 25 ítems con categoría de respuesta en escala de Likert. Su confiabilidad se determinó con el coeficiente alfa de Cronbach, el resultado fue 0,91. Como resultado se evidenció la satisfacción en la calidad del servicio educativo, precio, lugar, publicidad, promoción, relaciones públicas y la interacción de las plataformas educativas; actitud y aptitud del personal; tiempo de espera e instalaciones físicas. Se concluye que la mezcla de marketing alcanzó niveles de muy satisfactorio y satisfactorio, es bien valorada por los clientes, en las dimensiones producto, precio, plaza, promoción, personas, procesos 
y ambiente físico, lo que demuestra que se brinda un servicio educativo eficiente, para mantenerse en el mercado y tomar las decisiones de mejora en la educación.

Palabras clave: Mezcla del marketing, satisfacción del cliente, marketing educativo, producto, precio

\begin{abstract}
The objective of this research is to measure the level of customer satisfaction with respect to the marketing mix in the Unidad Educativa Particular Redemptio in the city of Jipijapa in the Province of Manabí. The research is exploratory and descriptive. The design was non-experimental and transectional. The population was 408 legal representatives of the Redemptio Private Educational Unit. The sample of 199 parents from the initial level to the unified general high school was calculated with a 5\% margin of error and 95\% confidence level, in the website of Asesoría Económica \& Marketing S. A. (2009). A 25-item questionnaire was used with a Likert scale response category. Its reliability was determined with Cronbach's alpha coefficient, this result was 0.91. As a result, satisfaction in the quality of the educational service, price, place, advertising, promotion, public relations and the interaction of educational platforms was evidenced; attitude and aptitude of staff; waiting time and physical facilities. It is concluded that most of the clients are satisfied in the variables of the product, price, place, promotion, people, processes and physical evidence related to the provision of the educational service.
\end{abstract}

Keywords: Marketing mix, customer satisfaction, educational marketing, product, price

\title{
Introducción
}

Las instituciones educativas del sector privado, para ofrecer calidad en los servicios educativos requieren proponer técnicas y metodologías para acercarse a su mercado objetivo. Las autoridades utilizan diversas estrategias de marketing para mantener la sostenibilidad y crecimiento de la entidad (Larreché, 2007). En el proceso de alcanzar los objetivos corporativos, se debe tener un plan que identifique fortalezas y debilidades de la unidad educativa, para dar respuesta a las necesidades de los clientes. El especialista en marketing considerando aspectos comerciales, sociales, bienes y servicios, combina de forma ordenada las cuatro P: producto, precio, plaza y promoción; y cualquier modificación a una de estas variables impactará la mezcla (Maubert, 2009). A esta mezcla, Hoang (2014) propone tres elementos adicionales: personas, procesos y evidencia física. El resultado es el modelo 7P en la comercialización de servicios. Estos servicios son recibidos por los clientes, que 
adquieren el bien para uso propio a un precio determinado por la empresa y aceptado socialmente (Bastos, 2006).

Es el cliente quien compra y asegura cifras para el crecimiento de la empresa (Trenzano \& Nadal, 1997). Estos criterios, confirman que el cliente es importante en todo negocio, que se planifican estrategias para cubrir sus necesidades y expectativas. El cliente actual, está capacitado, informado y exige precio pertinente y calidad del bien o servicio que recibe y una atención personalizada y directa. Los términos de atención y servicio son dos conceptos diferentes. La atención es la relación interpersonal amable y cordial y el servicio incluye los procesos, asesorías, ayuda e información que se brinda a un cliente. A veces se le ha dado más prioridad a la relación personal y se ha olvidado el objetivo final: su satisfacción (Kirberg \& González, 2010).

La satisfacción del cliente es un indicador que permite realizar retroalimentación sobre el giro del negocio, asumiendo nuevas estrategias que cubran y superen las expectativas de los clientes. Para Kotler \& Armstrong (2012), es la expresión del cumplimiento de las expectativas del consumidor por un producto, "Si el desempeño del producto no cubre las expectativas, el cliente se sentirá insatisfecho. Si el desempeño coincide con las expectativas, éste estará satisfecho. Si el desempeño excede las expectativas, se sentirá muy satisfecho o incluso encantado" (p.13).

También proporciona información respecto a los factores que influyen a esta variable (Eklöf y Westlund, 1998). Existen maneras de gestionar la satisfacción del cliente, pero, para que tenga relevancia, la empresa deberá determinar el valor añadido y el elemento diferenciador clave, lo que es una decisión estratégica del marketing (Domínguez, 2007). Por tanto, la satisfacción es una respuesta positiva que se deriva del encuentro entre el consumidor con un producto, e incluye un estado emocional que resulta de la evaluación del mismo y se aprecia de manera rentable en las cuentas de la entidad (Oliver, 1980; Westbrook, 1987; Kotler y Armstrong, 2008).

La satisfacción es un reflejo del rendimiento del servicio y ha sido empleado en educación como el instrumento para medir el rendimiento de una institución educativa (Blanco-Guillermety, 2004; Ham, 2003; Zineldin et al., 2011); es por esto, que la satisfacción en términos amplios, y no necesariamente la relacionada a la enseñanza recibida, debe estar directamente vinculada con la intención de recomendar el producto de manera positiva. La decisión del comprador por un producto está acompañada de la planificación de estrategias de marketing, "el mercadólogo debe encontrar la manera de cambiar el producto o de modificar las percepciones del consumidor" (Kotler y Armstrong, 2012 p. 154). 
El cliente satisfecho vuelve a comprar y recomienda la efectividad del producto. El cliente insatisfecho, por el contrario, deja de comprar, busca otra unidad educativa y desestima el producto que recibe. El reto del especialista en marketing es retener al cliente satisfecho y atraer a otros compradores, "el valor y la satisfacción de los clientes son bloques de construcción clave para desarrollar y administrar las relaciones con los clientes (Kotler y Armstrong, 2008, p. 8). Para garantizar la satisfacción de los clientes es importante ofrecerles valor, garantizando distinguirse de los competidores.

En las instituciones educativas se interactúa con clientes internos y externos. Para ello, es necesario el uso de herramientas de marketing, que contribuyan a mejorar el servicio y satisfacer la demanda. Al respecto Kotler y Armstrong (2012) plantean:

La mezcla de marketing es el conjunto de herramientas de marketing tácticas y controlables que la empresa combina para producir la respuesta deseada en el mercado meta. La mezcla de marketing incluye todo lo que la empresa puede hacer para influir en la demanda de su producto. Las muchas posibilidades pueden reunirse en cuatro grupos de variables conocidas como las cuatro P: producto, precio, plaza y promoción (p. 52).

Desde otra perspectiva, Hoang (2014) propone tres elementos adicionales: personas, procesos y evidencia física. El resultado es el modelo 7P en la comercialización de servicios (Lominé, Muchena, $\&$ Pierce, 2014). Este enfoque contribuye al análisis de la mezcla de la mercadotecnia en los servicios educativos. El marketing educativo es una estrategia de favor de un beneficio progresivo y debidamente entregado al cliente (Cecchio, Borrero, \& García, 2016).

La combinación de bienes y servicios que la empresa ofrece a sus clientes, es el producto Kotler y Armstrong (2008, 2012); para los autores, es un elemento esencial de la oferta de mercado general, en este sentido: "La planeación de la mezcla de marketing inicia con la formulación de una oferta que entregue valor a los clientes meta. Esta oferta se convierte en la base sobre la cual la empresa construye relaciones redituables con los clientes" (p. 224).

Los productos pueden ser bienes o servicios, los primeros pueden conservarse y su calidad es variada, mientras que los servicios son heterogéneos y los clientes obtienen experiencias distintas (Hoang, 2014). De este modo, la mezcla de marketing se orienta a ofrecer valor a sus clientes de manera rentable. El producto es mucho más que un objeto y los clientes compran satisfacciones, no productos. Es el conjunto de atributos físicos, de servicio y simbólicos que producen satisfacción o beneficios al usuario o comprador (Bello y Sains (2007). 
Por el producto se paga un precio y las ventas producen utilidades, a diferencia de los otros elementos que constituyen costos, en la mezcla de marketing se puede modificar con rapidez, lo que no ocurre con las características del producto (Kotler \& Armstrong, 2012). El precio es otra variable que sirve para determinar los ingresos de una empresa, es por ello, que se deben fijar considerando los costos, el mercado, proyecciones de recursos, rentabilidad y otros.

La institución planifica actividades planificadas para poner el producto a disposición de los consumidores meta (Kotler \& Armstrong 2008). En este sentido, el canal de marketing es el conjunto de organizaciones interdependientes que permiten que un producto o servicio llegue a manos del consumidor o usuario de negocios (Kotler \& Armstrong, 2012), en el momento y en lugar correcto y con una buena promoción. La mezcla de promoción sirve para comunicar información y valor para el cliente de forma persuasiva, acerca de los productos de una institución educativa. La promoción pretende lograr nuevos clientes y retener a los existentes (Lominé, Muchena, \& Pierce, 2014). El fin de la promoción es transmitir el mensaje apropiado con las bondades del producto, a los clientes meta, en el momento adecuado, genera compras.

La promoción carece de efectividad si no se cumplen con las expectativas de los clientes. Para ello, se requieren las siguientes herramientas de promoción: publicidad, es el pago de presentación y promoción de productos, por un patrocinador; promoción de ventas, son los incentivos que fomentan la compra o venta de un producto; ventas personales, representan la fuerza para vender y relacionarse con el cliente; relaciones públicas, con los diversos públicos para crear buena imagen corporativa; y, marketing directo, son las conexiones directas para obtener una respuesta inmediata y cultivar relaciones duraderas con el cliente (Kotler \& Armstrong, 2012).

Además, una estrategia de marketing de comunicación pertinente, en una institución educativa, requiere: identificar los requerimientos del destinatario de interés; diseñar mensajes que comuniquen obstáculos y fortalezas del producto y; evaluar la efectividad de la campaña de comunicación (Marcano, Fernández y Pérez (2009).

En este proceso, las personas representan un componente importante en la mezcla de marketing. La institución educativa, debe considerar habilidades y fortalezas de las personas, para tomar las decisiones respecto a la selección, reclutamiento, contratación y retención en el puesto de trabajo. Este aspecto es poco considerado en muchas ocasiones (Lominé, Muchena, \& Pierce, 2014). La presencia de excelente personal en la empresa contribuye a que se genere confianza en los clientes, buenas relaciones internas y externas. 
El personal de la institución es quien satisface las necesidades de los clientes, por estar en contacto directo con ellos; es necesario fomentar buenas relaciones y confianza con los clientes. También, se necesitan maestros apasionados por sus materias para brindar buena educación a los estudiantes. La efectividad del personal en la prestación de un servicio se puede medir con la apariencia, aptitudes y actitudes y eficiencia en las tareas y responsabilidades específicas (Hoang, 2014).

La forma en que se presta un servicio y según sus características puede ser complicado para demostrar los beneficios a los clientes. Para ello, se recurre a los procesos, que son las formas y procedimientos planificados para la entrega de un producto, (Lominé, Muchena \& Pierce,, 2014). Los procesos pueden incluir métodos de pago, tiempo de espera, servicios al cliente, atención postventa y procesos de entrega (Hoang, 2014). En este sentido, la evidencia física del producto representa el punto tangible y diferenciador observable. Es importante concentrarse en este elemento para competir, por ejemplo, los directivos de una escuela privada pueden proporcionar aulas bien diseñadas, pantallas gigantes, museos, bibliotecas y equipadas a diferencia de las públicas (Lominé, Muchena \& Pierce, 2014), (Hoang, 2014).

El presente artículo tiene por objetivo medir el nivel de satisfacción del cliente respecto de la mezcla del marketing en la Unidad Educativa Particular "Redemptio" de la ciudad de Jipijapa de la Provincia de Manabí.

\section{Definiciones del marketing educativo}

Además, de los constructos analizados, es preciso revisar varias definiciones del marketing educativo, se puede observar en el cuadro 1 .

\begin{tabular}{|l|l|l|}
\hline Autor & Definición & Características \\
\hline $\begin{array}{l}\text { Manes } \\
(1997)\end{array}$ & $\begin{array}{l}\text { Marketing educativo es el proceso de } \\
\text { investigación de las necesidades sociales tendiente } \\
\text { a desarrollar y llevar a cabo proyectos educativos } \\
\text { que las satisfagan. Su principal objetivo es } \\
\text { producir el desarrollo integral de las personas, a } \\
\text { través de servicios educativos sustentados en la } \\
\text { calidad y el mejoramiento continuo de la } \\
\text { organización educativa. }\end{array}$ & $\begin{array}{l}\text { Investigación de } \\
\text { necesidades sociales. } \\
\text { Mejoramiento continuo } \\
\text { de la organización } \\
\text { educativa }\end{array}$ \\
\hline Davis (1997) & $\begin{array}{l}\text { El marketing de servicios educativos, consiste en } \\
\text { el manejo de la relación entre las instituciones } \\
\text { educativas y sus clientes. En otras palabras, es el } \\
\text { medio mediante el cual la institución educativa } \\
\text { comunica su propósito, sus valores y servicios a } \\
\text { sus alumnos, a su propio personal, a los padres de }\end{array}$ & $\begin{array}{l}\text { Manejo de relación entre } \\
\text { instituciones educativas } \\
\text { y clientes. } \\
\text { Comunica su propósito a } \\
\text { la comunidad educativa. }\end{array}$ \\
\hline
\end{tabular}




\begin{tabular}{|c|c|c|}
\hline & $\begin{array}{l}\text { familia y a toda la comunidad educativa en } \\
\text { general. }\end{array}$ & \\
\hline $\begin{array}{l}\text { Alvarado } \\
(2003)\end{array}$ & $\begin{array}{l}\text { Es el cumplimiento de la acción comercial que } \\
\text { orienta el flujo del servicio, desde el colegio hacia } \\
\text { los consumidores del servicio para satisfacer } \\
\text { necesidades. En la calidad educativa conjugan la } \\
\text { calidad de promesa y calidad de demanda. }\end{array}$ & $\begin{array}{l}\text { En la calidad educativa } \\
\text { conjugan la calidad de } \\
\text { promesa y calidad de } \\
\text { demanda. }\end{array}$ \\
\hline $\begin{array}{l}\text { Manes } \\
(2004)\end{array}$ & $\begin{array}{l}\text { El marketing educativo es el proceso de } \\
\text { investigación de las necesidades sociales, para } \\
\text { desarrollar servicios educativos tendientes a } \\
\text { satisfacerlas, acordes a su valor percibido, } \\
\text { distribuidos en tiempo y lugar, y éticamente } \\
\text { promocionados para generar bienestar entre } \\
\text { individuos y organizaciones }\end{array}$ & $\begin{array}{l}\text { Proceso de investigación } \\
\text { de necesidades sociales. } \\
\text { desarrollar servicios } \\
\text { educativos, acorde a su } \\
\text { valor percibido, } \\
\text { distribuido en tiempo y } \\
\text { lugar }\end{array}$ \\
\hline $\begin{array}{l}\text { American } \\
\text { Marketing } \\
\text { Association } \\
(2007)\end{array}$ & $\begin{array}{l}\text { Es la actividad, grupo de instituciones y procesos } \\
\text { para crear, comunicar, distribuir e intercambiar } \\
\text { ofrecimientos que tengan valor para los } \\
\text { consumidores, clientes, partners y sociedad en } \\
\text { general. }\end{array}$ & $\begin{array}{l}\text { Crear, comunicar, } \\
\text { distribuir e intercambiar } \\
\text { ofrecimientos que tengan } \\
\text { valor para los } \\
\text { consumidores }\end{array}$ \\
\hline $\begin{array}{l}\text { Nuñez } \\
\text { (2017) }\end{array}$ & $\begin{array}{l}\text { El marketing educativo camina entre el marketing } \\
\text { empresarial y marketing social, pues aunque un } \\
\text { centro sea privado y persiga el fin último y } \\
\text { legítimo de obtener beneficios, siempre } \\
\text { desarrollará una labor social de primer orden, la } \\
\text { más importante, bajo mi punto de vista: educar a } \\
\text { las generaciones futuras. }\end{array}$ & $\begin{array}{l}\text { Camina entre el } \\
\text { marketing empresarial y } \\
\text { marketing social, para } \\
\text { desarrollar una labor } \\
\text { social de primer orden }\end{array}$ \\
\hline
\end{tabular}

Cuadro 1. Definiciones de marketing educativo

De estas definiciones se pueden decir que el marketing educativo: tiene carácter social y económico, su propósito es comunicar a los clientes, desarrollar servicios educativos con valor y busca retener alumnos clientes. Se puede definir al marketing educativo como una herramienta clave para las instituciones educativas porque permite fijar sus objetivos orientados a la retención y captación de nuevos estudiantes de manera rentable y con beneficio social.

El marketing educacional pretende los siguientes objetivos: mejorar la percepción general y generar una buena opinión pública; despertar el interés de la institución en un grupo objetivo; orientar estratégicamente la institución hacia al mercado potencial; utilizar las relaciones, el contacto y la tecnología para vínculos permanentes; asignar eficientemente los recursos; medir los resultados de la gestión educativa y empresarial; aportar al desarrollo social. Con una planificación estratégica adecuada, ciertamente, se mejora la posición competitiva en el mercado de la educación dándole valor a la marca corporativa (Bustos y Becker,2009). 


\section{Materiales y métodos}

El alcance de la investigación es de tipo descriptivo y correlacional, el diseño de investigación fue no experimental transversal. La población estuvo compuesta por 408 representantes legales, padres de familia, de la Unidad Educativa Particular Redemptio. La muestra fue de 199 padres de familia desde el nivel inicial hasta el bachillerato general unificado, se calculó con el 5\% de margen de error y el 95\% de nivel de confianza, en el sitio web de Asesoría Económica \& Marketing S. A. (2009). Se utilizó un cuestionario de 25 ítems organizados por las siete P del marketing (Hoang,2014): producto, precio, plaza, promoción, personas, procesos y evidencia física, con categorías de respuesta en escala de Likert (4 Muy satisfecho, 3 Satisfecho, 2 Poco insatisfecho, 1 Insatisfecho). Su confiabilidad se determinó con el coeficiente alfa de Cronbach analizado en el programa SPSS versión 23; este resultado fue de 0,91 , lo que permitió asegurar que el instrumento era confiable.

\section{Resultados}

Los resultados de la aplicación del cuestionario, se tabularon en función de las dimensiones de la herramienta mezcla de marketing. En lo relativo a la dimensión de productos, se consideraron los indicadores: servicio, variedad y garantías del producto. Tabla 2.

\begin{tabular}{|c|c|c|c|c|c|}
\hline \multirow[t]{2}{*}{ Dimensión: Producto } & \multicolumn{4}{|c|}{ Satisfacción \% } & \multirow[b]{2}{*}{ total } \\
\hline & $\begin{array}{l}1 \\
\mathrm{I}\end{array}$ & $\begin{array}{c}2 \\
\text { PS }\end{array}$ & $\begin{array}{l}3 \\
\mathrm{~S}\end{array}$ & $\begin{array}{c}4 \\
\mathrm{MS}\end{array}$ & \\
\hline \multicolumn{6}{|l|}{ Servicio } \\
\hline 1. La calidad del servicio educativo en general. & 2 & 5 & 56 & 37 & 100 \\
\hline 2. Las metodologías empleadas en el proceso de aprendizaje. & 0 & 15 & 46 & 39 & 100 \\
\hline 3. Nivel de actualización y utilidad de los temas del plan de estudios. & 0 & 7 & 43 & 50 & 100 \\
\hline 4. Los valores que fomenta la Unidad Educativa. & 0 & 2 & 32 & 66 & 100 \\
\hline \multicolumn{6}{|l|}{ Variedad } \\
\hline 5. La variedad de las asignaturas que brinda la Unidad Educativa. & 7 & 7 & 38 & 48 & 100 \\
\hline $\begin{array}{l}\text { 6. La innovación de recursos didácticos en la prestación del servicio } \\
\text { educativo. }\end{array}$ & 0 & 12 & 40 & 48 & 100 \\
\hline \multicolumn{6}{|l|}{ Garantías } \\
\hline 7. Las condiciones de seguridad y protección dentro de la UE & 0 & 1 & 29 & 70 & 100 \\
\hline
\end{tabular}

Tabla 2. Dimensión Producto: Servicio, variedad, garantías

En lo concerniente a los padres de familia, se observa que están satisfechos por la calidad del servicio educativo en general y las metodologías empleadas. Están muy satisfechos con el nivel de actualización y utilidad de los temas del plan de estudios; así como, los valores que fomenta la unidad educativa. 
En lo relativo a la dimensión precio, se consideraron los indicadores precio de lista y período de pago. Tabla 3

\begin{tabular}{|c|c|c|c|c|c|}
\hline \multirow[t]{3}{*}{ Dimensión: Precio } & \multicolumn{4}{|c|}{ Satisfacción \% } & \multirow[b]{2}{*}{ Total } \\
\hline & 1 & 2 & 3 & 4 & \\
\hline & I & PS & $\mathrm{S}$ & MS & \\
\hline \multicolumn{6}{|l|}{ Precio de lista } \\
\hline $\begin{array}{l}\text { 8. Los precios que cobra la Unidad Educativa por el servicio } \\
\text { educativo. }\end{array}$ & 3 & 32 & 45 & 20 & 100 \\
\hline \multicolumn{6}{|l|}{ Precio de pago } \\
\hline 9. La accesibilidad de los periodos de pago. & 3 & 23 & 53 & 21 & 100 \\
\hline
\end{tabular}

Tabla 3 Dimensión Precio: precio de lista, periodo de pago

Los resultados de la Tabla 3 muestran que existe nivel de satisfacción respecto de los precios que se cobran por los servicios educativos y la accesibilidad de los periodos de pago. Se puede decir, que la dimensión precio, está bien valorada y la unidad educativa se ha preocupado por ofrecer un servicio educativo coherente con el precio.

En lo concerniente a la dimensión plaza, se consideró el indicador ubicación de la institución. Tabla 3.

\begin{tabular}{|c|c|c|c|c|c|}
\hline \multirow[t]{2}{*}{ Dimensión: Plaza } & \multicolumn{4}{|c|}{ Satisfacción \% } & \\
\hline & $\begin{array}{l}1 \\
\text { I }\end{array}$ & $\begin{array}{c}2 \\
\text { PS }\end{array}$ & $\begin{array}{l}3 \\
S\end{array}$ & $\begin{array}{c}4 \\
\text { MS }\end{array}$ & $\begin{array}{c}\text { Tota } \\
1\end{array}$ \\
\hline \multicolumn{5}{|l|}{ Ubicación } & \\
\hline 10. El lugar en que está ubicada la Unidad Educativa. & 1 & 7 & 40 & 52 & 100 \\
\hline
\end{tabular}

Tabla 3. Dimensión Plaza: ubicación

La Tabla 3 muestra que los padres de familia están muy satisfechos por el lugar en que está ubicada la Unidad Educativa. Se puede decir, que esta dimensión, se encuentra muy bien valorada, por cuanto, se evidencia accesibilidad y facilidades de ingreso.

En la dimensión promoción, se consideraron los indicadores publicidad, promoción en ventas, relaciones públicas y marketing directo. Tabla 4

\begin{tabular}{|l|l|l|l|l|l|}
\hline Dimensión: Promoción & \multicolumn{3}{|l|}{ Satisfacción \% } & \\
\cline { 2 - 7 } & $\begin{array}{c}1 \\
\text { I }\end{array}$ & $\begin{array}{c}2 \\
\text { PS }\end{array}$ & S & MS & Total \\
\hline Publicidad & & & & & \\
\hline 11. Los canales de comunicación utilizados por la U. Educativa & 1 & 15 & 50 & 34 & 100 \\
\hline 12. Los anuncios publicitarios utilizados por la U. Educativa & 1 & 10 & 62 & 27 & 100 \\
\hline
\end{tabular}




\begin{tabular}{|c|c|c|c|c|c|}
\hline \multicolumn{5}{|l|}{ Promoción en ventas } & \\
\hline 13. Las recompensas/incentivos por ser cliente habitual. & 6 & 23 & 42 & 29 & 100 \\
\hline 14. Las becas otorgadas por la unidad educativa. & 6 & 24 & 45 & 25 & 100 \\
\hline \multicolumn{5}{|l|}{ Relaciones públicas } & \\
\hline 15. Los eventos especiales realizados por la u. Educativa & 2 & 15 & 62 & 21 & 100 \\
\hline $\begin{array}{l}\text { 16. La selección de tópicos impartidos en } \\
\text { conferencias/talleres/reuniones. }\end{array}$ & 2 & 7 & 55 & 36 & 100 \\
\hline \multicolumn{5}{|l|}{ Marketing directo } & \\
\hline $\begin{array}{l}\text { 17. La interacción a través de plataformas educativas, página } \\
\text { web, redes sociales y otros. }\end{array}$ & 5 & 14 & 57 & 24 & 100 \\
\hline
\end{tabular}

Tabla 4. Dimensión Promoción: Publicidad, promoción en ventas, relaciones públicas, marketing directo

La Tabla 4, muestra que los padres de familia expresaron su nivel de satisfacción, respecto de la publicidad, canales de comunicación y anuncios publicitarios utilizados por la Unidad Educativa para ofertar y transmitir los aspectos positivos del producto y servicios educativos. De igual manera, se sienten atendidos, por la promoción en ventas, recompensas e incentivos por ser cliente habitual y las becas otorgadas a estudiantes con buen rendimiento académico y que requieren ayuda económica.

En la dimensión personas, se consideraron los indicadores apariencia física, aptitudes, actitudes y eficiencia. Tabla 5.

\begin{tabular}{|c|c|c|c|c|c|}
\hline \multirow[t]{2}{*}{ Dimensión: Personas } & \multicolumn{4}{|c|}{ Satisfacción \% } & \multirow[b]{2}{*}{ Total } \\
\hline & $\begin{array}{l}1 \\
\mathrm{I}\end{array}$ & \begin{tabular}{|l|l|l}
2 \\
PS
\end{tabular} & $\begin{array}{l}3 \\
S\end{array}$ & MS & \\
\hline \multicolumn{5}{|l|}{ Apariencia física } & \\
\hline $\begin{array}{l}\text { 18. La apariencia física con respecto al uso del uniforme de los } \\
\text { docentes. }\end{array}$ & 0 & 4 & 38 & 58 & 100 \\
\hline \multicolumn{5}{|l|}{ Aptitudes } & \\
\hline 19. El nivel de capacitación del personal de la Unidad Educativa. & 0 & 4 & 42 & 54 & 100 \\
\hline \multicolumn{5}{|l|}{ Actitudes } & \\
\hline 20. La actitud demostrada por el personal de la Unidad Educativa. & 0 & 15 & 37 & 48 & 100 \\
\hline \multicolumn{5}{|l|}{ Eficiencia } & \\
\hline $\begin{array}{l}\text { 21. La eficiencia del servicio educativo ofrecido por las personas: } \\
\text { autoridades, docentes, personal administrativo. }\end{array}$ & 2 & 5 & 49 & 44 & 100 \\
\hline
\end{tabular}

Tabla 5. Dimensión Personas: apariencia física, aptitudes, actitudes, eficiencia

En la Tabla 5, se observa que los padres de familia valoran como muy satisfactoria la apariencia física, con respecto al uso de uniformes de los docentes. En las aptitudes, se aprecia el nivel de capacitación del personal docente; los clientes consideran muy satisfactoria la actitud demostrada por 
el personal. Es satisfactorio el nivel de eficiencia del servicio educativo ofrecido por las personas: autoridades, docentes, personal administrativo.

En la dimensión procesos se consideró el indicador tiempo de espera.Tabla 6

\begin{tabular}{|c|c|c|c|c|c|}
\hline \multirow[t]{2}{*}{ Dimensión: Procesos } & \multicolumn{4}{|c|}{ Satisfacción \% } & \multirow[b]{2}{*}{ Total } \\
\hline & $\begin{array}{l}1 \\
\mathrm{I}\end{array}$ & $\begin{array}{l}2 \\
\text { PS }\end{array}$ & $\begin{array}{l}3 \\
S\end{array}$ & $\begin{array}{l}4 \\
M S\end{array}$ & \\
\hline \multicolumn{5}{|l|}{ Tiempo de espera } & \\
\hline $\begin{array}{l}\text { 22. Los tiempos de espera en los procesos de matrícula, } \\
\text { entrega de libretas, atención a padres de familia, actividades } \\
\text { culturales, deportivas, entre otros. }\end{array}$ & 7 & 12 & 48 & 33 & 100 \\
\hline
\end{tabular}

Tabla 6. Dimensión Procesos: Tiempo de espera

La Tabla 6, evidencia satisfacción de los padres de familia, por la forma en que se prestan los servicios, y los beneficios que reciben. Estos procesos incluyen los métodos de pago, el tiempo de espera, entrega de las libretas, atención en las inquietudes y necesidades que tienen como clientes. Consideran que las actividades culturales, deportivas, sociales, se desarrollan oportuna y pertinente.

En la dimensión ambiente físico, se consideraron los indicadores instalaciones e infraestructura. Tabla 7.

\begin{tabular}{|l|l|l|l|l|l|l|}
\hline Dimensión: Ambiente físico & \multicolumn{3}{|l|}{ Satisfacción \% } & \\
\cline { 2 - 6 } & \multicolumn{1}{|l|}{$\begin{array}{l}2 \\
\text { I }\end{array}$} & $\begin{array}{l}3 \\
\text { S }\end{array}$ & $\begin{array}{l}4 \\
\text { Ms }\end{array}$ & Total \\
\hline Instalaciones & & & \\
\hline $\begin{array}{l}\text { 23. La limpieza y orden de los recursos físicos de la unidad } \\
\text { educativa. }\end{array}$ & 0 & 8 & 35 & 57 & 100 \\
\hline Infraestructura & 2 & 14 & 52 & 32 & 100 \\
\hline 24. El estado físico de las aulas & 2 & 5 & 50 & 43 & 100 \\
\hline $\begin{array}{l}\text { 25. El estado físico de las canchas deportivas, laboratorios, } \\
\text { biblioteca, otras instalaciones de la Unidad Educativa. }\end{array}$ & & & & \\
\hline
\end{tabular}

Tabla 7. Dimensión Ambiente Físico: Instalaciones, infraestructura

La Tabla 7 muestra los resultados, respecto de la dimensión ambiente físico, se valora como muy satisfactoria la limpieza y orden de los recursos físicos de la Unidad Educativa. Los padres de familia consideran que es satisfactoria la infraestructura de la institución, por el estado físico de las aulas, canchas deportivas, laboratorios, biblioteca y otras instalaciones.

\section{Discusión}


La investigación pretendió determinar la satisfacción de los clientes, respecto del servicio que ofrece, en este sentido, la variedad de asignaturas y la innovación de recursos didácticos utilizados; y, las garantías de las condiciones de seguridad y protección dentro de la entidad llegaron al nivel de muy satisfactorio. Estos resultados concuerdan con el estudio de los autores Martínez, Almanza, Ustate, (2018), quienes demostraron la satisfacción de los estudiantes respecto a sus necesidades de formación profesional en la Universidad de La Guajira Colombia.

En lo referente al precio es coherente con el servicio educativo, sin descuidar los ingresos de la institución y considerando costos, mercado, proyecciones de recursos, entre otros. Estos elementos son relevantes desde el aspecto económico, contribuyen al crecimiento empresarial; no obstante, la entidad también se preocupa por el fin social de su plan de marketing educativo. Estos resultados son contrarios al estudio de Briceño (2018), porque se determinó que la dimensión precio fue valorada como regular. En cambio, en la investigación de Flores, Erreyes y Mayorga (2017), se identificó que la empresa fija sus precios de acuerdo con las decisiones administrativos, a los márgenes de utilidad y a los costos. Por otra parte, en el estudio de Córdova y Moreno (2017), se determinó que una empresa para estar al nivel de sus competidores, entregando servicios de alto valor agregado, requiere de una buena estrategia de fijación de precios que sean atractivas para el cliente.

Se ofrece una planificación adecuada, un servicio accesible al cliente y un lugar que permite facilidad para ingresar y salir de la entidad. Estos resultados, son coherentes con la investigación de Dalongaro, (2014), en la que se determinó que los clientes estaban satisfechos por el lugar donde se encontraban el establecimiento. La localización de la entidad, influye en el cliente al recomendar a otros los beneficios del producto convirtiéndose en un elemento de importancia estratégica para la organización educativa.

Respecto a las relaciones públicas, eventos especiales, selección de tópicos impartidos en conferencias, talleres y reuniones; interacción a través de plataformas educativas, página web y redes sociales; la unidad educativa se preocupa por dar a conocer las características de servicio. En la investigación de Fernández, (2015) se concluye que el marketing mix es fundamental porque devela un especial interés por las acciones de la promoción en la estructura de la oferta de servicios. Por otra parte, los autores Abrigo, Celi, Briceño, \& Ruth, (2020), manifiestan en la investigación del marketing mix en los emprendimientos universitarios que, con el uso de las redes sociales como Facebook, Instagram y Youtube, se da a conocer un producto. Además, en la investigación de Perdigón, Viltres y Madrigal, (2018), se determinó que la promoción también se puede aplicar de manera digital. 
La institución se preocupa por mantener actualizados y capacitados a los docentes y personal administrativo de la institución, lo que redunda en una educación de calidad. En el estudio de Zárraga, Molina y Corona (2018), se determinó que la eficiencia con que opera el personal de una organización genera satisfacción en los consumidores. Además, en la investigación de Santistevan, Loor y Cano (2020) se evidenció que es importante tener claro los objetivos de la empresa por parte de los colaboradores para que estos puedan ejercer sus funciones con efectividad. La presencia de excelente personal en la empresa genera confianza en los clientes y fortalece las relaciones internas y externas. En este proceso de oferta y recepción de los productos, es el personal docente y administrativo, que tiene más contacto con los clientes.

La unidad educativa tiene un plan de marketing que equilibra los procesos, actividades y programación internas, considerando la disponibilidad de tiempo, recursos económicos, relaciones familiares y sociales de los clientes. Los padres pueden compartir y participar de la educación de sus hijos con sus otras actividades y se fortalece el proceso de educación. En la investigación de Arriaga, Ávalos y Martinez, (2017), se demostró que, para incrementar el potencial y el rendimiento de una empresa se debe garantizar el control de procesos en todas áreas de la organización.

Los ambientes de aprendizaje son importantes en el desarrollo y formación de los estudiantes, contribuyen para alcanzar los resultados de aprendizaje. En el estudio de Maché \& Frías (2016) también se hace referencia a que el ambiente físico es un aspecto visualmente atractivo por el cliente, siendo una variable con mayor valor percibido. Las entidades deben garantizar ambientes agradables y cómodos, acceso a recursos bibliográficos, porque, la evidencia física del producto representa el punto tangible y diferenciador observable, que el cliente valora para escoger los servicios educativos.

\section{Conclusiones}

La mezcla de marketing en la Unidad Educativa Particular Redemptio, ha alcanzado niveles de muy satisfactorio y satisfactorio, es bien valorada por los clientes, en las dimensiones producto, precio, plaza, promoción, personas, procesos y ambiente físico, lo que demuestra que se brinda un servicio educativo eficiente, para mantenerse en el mercado y tomar las decisiones de mejora en la educación. El equilibrio en la mezcla de marketing contribuye a la fidelidad del cliente muy satisfecho, para que sea leal al servicio que le ofrece la entidad, tiene como objetivo conseguir clientes y obtener ingresos económicos para la empresa; el otro fin es el aporte social que se debe evidenciar en el producto que se ofrece y que el consumidor valora para preferirlo. 
El producto en las instituciones educativas juega un rol fundamental porque los clientes compran satisfacciones, por el conjunto de atributos físicos, simbólicos y de servicio que representan las actividades educativas que reciben.

El precio representa el valor monetario que favorece a las entidades educativas con fines de lucro, porque generan utilidades por el servicio que ofrecen a los clientes; el marketing influye en este precio, lo puede modificar debido a los costos, proyecciones y rentabilidad.

El punto de venta o plaza permite poner los productos educativos a disposición de la comunidad educativa o usuarios de los servicios; la mezcla de marketing aporta con la planificación para que dicho producto se ofrezca en el momento y lugar correcto para satisfacer las expectativas del consumidor.

La mezcla de promoción sirve a las organizaciones educativas para comunicar de forma convincente, la información y valor acerca de los productos de educación. La promoción que planifica un buen mensaje, que declara las bondades de los servicios educativos, logrará nuevos clientes y retiene a los existentes, de tal forma, que se genera compras e incremento de utilidades.

Las personas representan las habilidades y fortalezas en la mezcla de marketing para una institución educativa; la selección de excelente y el trabajo eficiente del personal influye en la confianza y relaciones con los clientes internos y externos. En una entidad de educación, se requieren maestros con vocación y pasión para enseñar y brindar un servicio de calidad que satisfaga las necesidades de una educación integral.

\section{Bibliografía}

Abrigo, I., Celi, Y., Briceño, C., \& Ruth, O. (2020). Valoración del marketing mix en los emprendimientos universitarios. Dialnet, 6(10). 326-344. Recuperado de: https://dialnet.unirioja.es/servlet/articulo?codigo=7389064.

American Marketing Association (2007). Asociación Americana de Marketing. Definición Arriaga, F., Ávalos, D., \& Martinez, E. (2017). Propuesta de estrategis de mejora basadas en análisis FODA en las pequeñas emrpesas de Arandas, Jalisco, México. Ra Ximhai, 13(3), 417-424. Recuperado de: https://www.redalyc.org/pdf/461/46154070025.pdf.

Bastos, A. I. (2006). Fidelización del Cliente. Vigo-España: Ideas propias Editorial.

Bello, J. L. y Sainz, A. (2007) Claves para gestionar precio, producto y marca. Cómo afrontar una guerra de precios. Colección e directivos. 
Cecchio, D., Borrero, T., \& García, J. P. (2016). Marketing educativo como estrategia de negocio en universidades privadas. Revista de la facultad de ciencias económicas, administrativas y contables de la Universidad de Simón Bolívar, 9(1), 160-175.

Córdova, C., \& Moreno, D. (2017). Importancia de una buena estrategia de fijación de precios como herramienta de penetración de mercados. Revista de la Facultad de Ciencias Económicas y Administrativas de la Universidad de Nariño, 18(2), 58-68. Recuperado de: https://revistas.udenar.edu.co/index.php/rtend/article/view/3667/4315.

Dalongaro, R. (2014). La satisfacción del cliente con el supermercadismo en áreas de frontera. Ciencias Administrativas, 4, 35-49. Recuperado de: https://www.redalyc.org/pdf/5116/511651380005.pdf.

Davis, B. (1997). Strategic marketing for schools: how to harmonise marketing and strategic development for an effective school. Pearson Education. London, England

Fernández, V. (2015). Marketing mix de servicios de información. Bibliotecas. Anales de investigación, 11 64-78. Recuperado de: https://dialnet.unirioja.es/servlet/articulo?codigo=5704542.

Flores, J., Erreyes, H., \& Mayorga, M. (2017). Costos de producción y fijación de precios en empresas artesanales. Caso de estudio: DAYANTEX. Revista Publicando, 4(12), 541-553. https://core.ac.uk/download/pdf/236644985.pdf.

González, M., Frias-Jiménez, R., Gómez, O. (2016). Análisis de la calidad percibida por el cliente en la actividad hotelera. Ingeniería Industrial, 37(3), 253-265. Recuperado de: http://scielo.sld.cu/pdf/rii/v37n3/rii040316.pdf.

Hoang, P. (2014). Business Management. Australia: IBID PRESS.

Kirberg, A. S., \& González, D. S. (2010). Marketing para Emprendedores. Bogotá: ECOE Ediciones

Kotler, P., \& Armstrong, G. (2008). Fundamentos de marketing. México: Pearson Educación.

Kotler, P., \& Armstrong, G. (2012). Marketing. México: PEARSON.

Larreché, J. M. (2007). Administración del marketing. México: Mc Graw Hill

Lominé, L., Muchena, M., \& Pierce, R. (2014). Bussines Management. United Kingdom: OXFORD.

Manes, J. M. (2004). Marketing para instituciones educativas. Guía para planificar la captación y retención de alumnos. Buenos Aires: Granica.

Marcano, Y., Fernández, G., \& Pérez, D. (2009). Diagnóstico de la mezcla de promoción de los programas de posgrado en las universidades públicas del estado Falcón. Revista Venezolana de Gerencia, 14(47), 429-445.

Martínez, L., Almanza, K., \& Ustate, M. (2018). Marketing educativo como factor de competitividad en las instituciones de educación superior. Revista Boletin Redipe, 7(10), 205-215. 
Maubert, C. G. (2009). Fundamentos de Marketing. México: Pearson.

Nuñez, V. (2017) Marketing educativo. Cómo comunicar la propuesta de valor de nuestro centro. Innovación Educativa.

Perdigón, R., Viltres, H., \& Madrigal, I. (2018). Estrategias de comercio electrónico y marketing digital para pequeñas y medianas empresas. Revista Cubana de Ciencias Informáticas, 12(3), 192-208. Recuperado de: http://scielo.sld.cu/pdf/rcci/v12n3/rcci14318.pdf.

Trenzano, J. M., \& Nadal, J. F. (1997). La conducta del Consumidor y del Cliente. Madrid: Ediciones Díaz de Santos, S. A.

Santistevan, J., Loor, H., \& Cano, E. (2020). Cuadro de Mando Integral desde la perspectiva aprendizaje y crecimiento en el sector de alimentos balanceados en Ecuador. Dominio de las ciencias, 6(5), 179-191. Recuperado de: https://www.dominiodelasciencias.com/ojs/index.php/es/article/view/1594.

Zárraga, L., Molina, V., \& Corona, E. (2018). La satisfacción del cliente basada en la calidad del servicio a través de la eficiencia del personal y eficiencia del servicio: un estudio empírico de la industria restaurantera. RECAI Revista de Estudios en Contaduría, Administración e Informática, 7(18), 46-65. Recuperado de: https://recai.uaemex.mx/article/view/9268. 